\title{
Focused Ion Beam Structuring of Photonic Components and Sensors
}

\author{
Philipp M. Nellen, Rolf Brönnimann \\ Empa, Materials Science and Technology, Laboratory Electronics/Metrology \\ CH-8600 Dübendorf, Switzerland, T. +41 182343 53, F: +41182340 54, philipp.nellen@empa.ch
}

\begin{abstract}
This paper discusses direct focused ion beam (FIB) writing of microstructured photonic elements and sensors like apertures on tips of optical fibers, microlenses, or photonic crystals. Emphasis is mainly on the fabrication side.
\end{abstract}

Keywords: Focused Ion Beam Structuring, Photonic Components, Sensors

\section{Introduction}

Direct microstructuring of photonic elements and sensors is an attractive way for miniaturization. One among other tools is the focused ion beam (FIB) technology. FIB technology was commercialized in the early $80^{\text {iics }}{ }^{[1]}$. First applications were in microelectronics reliability and failure analysis mainly taking advantage of the easy way to prepare crosssections and TEM-lamellae. Nowadays, with the rapid advance in micro- and nanotechnology new FIB applications appear. Modern FIB systems are used for probe preparation of materials with a wide range of properties, for structural modifications by gas assisted deposition of various materials, and for machining and milling structures in various materials by sputtering or by gas assisted etching. FIB structure writing and patterning uses the fact that focused high energy ions sputter away material or activate reactive gases for etching or deposition. However, the amount of processed material depends on various parameters in a nonlinear way.

Predictable microstructuring with FIB may result in new applications like MOEMS device fabrication and modification, scanning probe (optical) microscope tips, micromedical device, micro- and nano-print master fabrication (e.g. diffractive optical elements), photonic elements and sensors, and others. The paper discusses microstructuring ${ }^{[2]}$ and shows first promising examples (microlens and fiber optical aperture) demonstrating feasibility for optical applications.

\section{Instrumentation}

The microstructuring experiments were made with a dual column FIB/SEM instrument (FEI 235) with 30kV Ga ${ }^{+}$ions.

Optical experiments with modified optical fiber tips were performed with a semiconductor laser of $650 \mathrm{~nm}$ for alignment, and with a non-coherent light source with a wavelength of $\lambda=840 \mathrm{~nm}$. A camera was used to measure patterns in transmission, either by focusing directly on the fiber tip with help of a microscope objective or by examining at the diffraction pattern in the far field.

\section{Microstructuring of lenses}

A FIB system typically has a beam deflection unit, which allows the ion beam to be focused on individual pixels for a specified dwelltime. Pixel size depends on the chosen magnification, whereas the ion beam diameter depends on the actual ion current and a proper focusing.

First patterns with dots and lines were milled in silicon to observe ion beam shape and milling behavior for various distances and dwelltimes. At an ion current of $130 \mathrm{pA}$ the ion beam shape is assumed to be Gaussian with $\sigma=8.5 \mathrm{~nm}$ according to instruments specification. A straight forward application for dots and holes may be photonic crystal fabrication either directly or as masks for etching procedures. However, effects like material redeposition and selffocusing have to be considered ${ }^{[3]}$.

In a next experiment squares were milled with different strategies in order to study and optimize milling. Each square was written with 312 lines of 311 dots. The distance was chosen to be $3 / 2 \sigma$, ion current was $130 \mathrm{pA}$. The magnification was adjusted in a way that the smallest increment (pixel size) of the beam deflection unit corresponds to $\sigma / 2$. The dose was always $0.8 \mathrm{fC} / \mathrm{nm}^{2}$. One set of squares was written in a single pass with a dwell time of $1 \mathrm{~ms} / \mathrm{dot} / \mathrm{pass}$ and the second set in 100 passes with a dwell time of $0.01 \mathrm{~ms} /$ dot/pass. The squares were written with vertical lines. In Figs. 1a and $1 \mathrm{~b}$

17th International Conference on Optical Fibre Sensors, Marc Voet, Reinhardt Willsch, 
the sequences written symmetrically from the edges to the middle are show first with the single pass (a) and second with the 100 passes (b). The lower part shows the corresponding cross sections. For the multiple passes the results are nearly independent from the writing strategies and flat. In the single pass cases, a strong dependence on the writing strategy is obvious. Material must have been redeposited and possibly also a self-focusing effect by reflected grazing incident ions caused a deeper milling. The case of the single pass with lines alternatively written from one edge to the middle is discussed further in detail.

A milling sequence (cross sections) with increasing number of milled lines (from the edges to the center) is shown in Fig. 1c. An average sputter efficiency of 6 atoms per incident ion and an average milling efficiency (without the redeposited material) of 2.4 atoms per incident ion were found. The maximum milled depth was found to increase with the width of the milled area, although total dose was always $0.8 \mathrm{fC} / \mathrm{nm}^{2}$. The milled area width is linear with the number of lines with a slope of $12.5 \mathrm{~nm}$, which corresponds to the chosen pixel spacing. Also the width of the ridge decreases linearly with the number of milled lines, as expected.

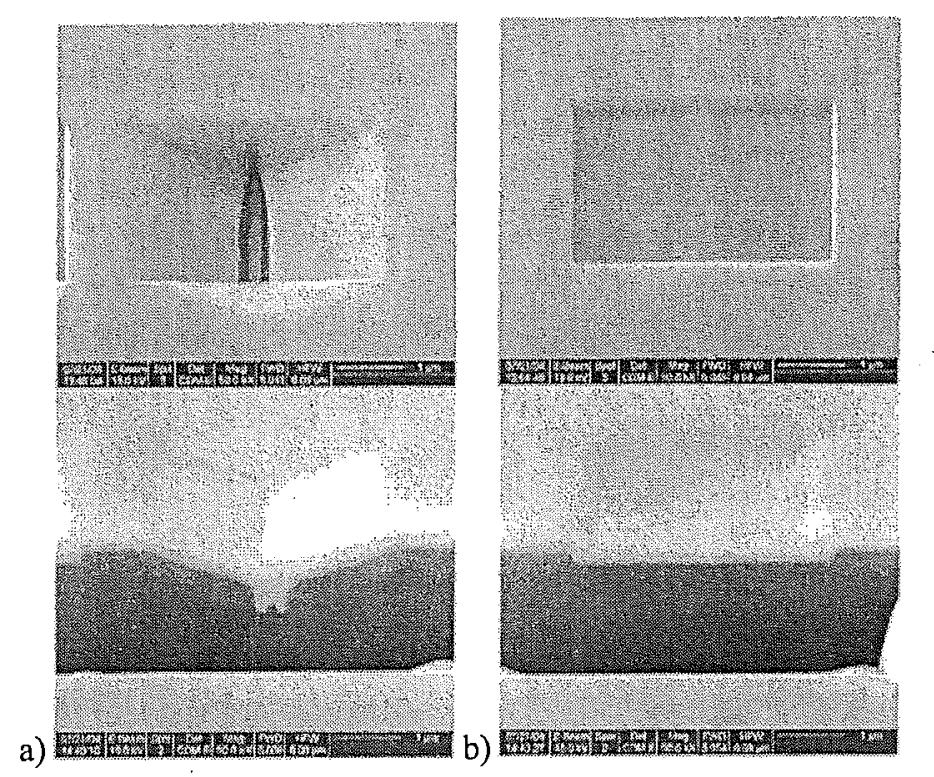

Fig. 1. Square, milled in a single pass (a) and in 100 passes (b), top: $52^{\circ}$-view, bottom: cross sections; (c) milling sequence of (a) with increasing number of milled lines $(5,60,120,145,152,156)$.

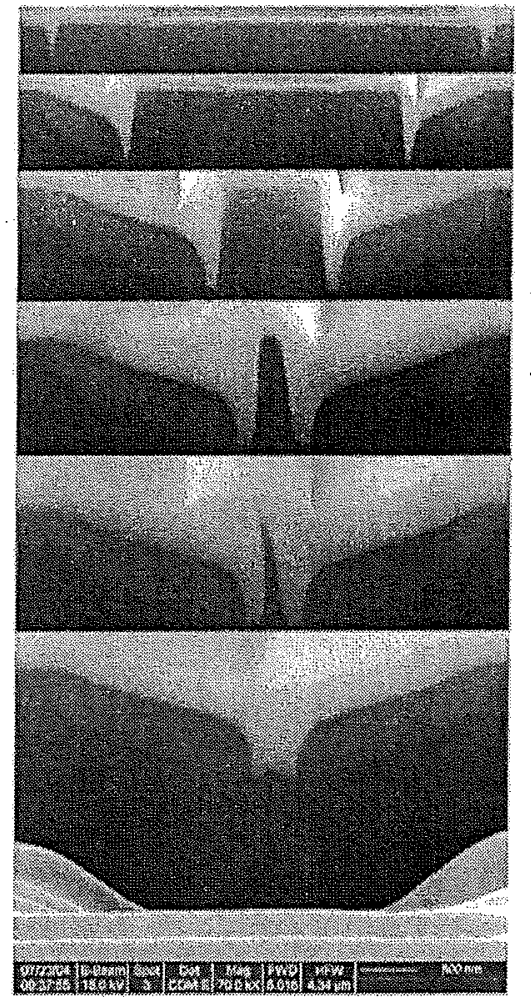

c)

The example in Fig. 2a is a first test to write Fresnel lens patterns (focal length $f=5 \mu \mathrm{m}$, wavelength of $\lambda=840 \mathrm{~nm}$ ). This Fresnel structure was milled in concentric circles (not line after line) from the center outwards. Ion beam current was $134 \mathrm{pA}$ and maximum dwell time was $36.2 \mu \mathrm{s}$. The dwell times were simply chosen to be proportional to the theoretical Fresnel pattern. The pattern was repetitively milled 120 times. The achieved depth is a factor of 6 smaller than that of the calculated Fresnel lens but shows a good correspondence (the theoretical curve in Fig. $2 \mathrm{~b}$ is scaled down by a factor of 6). However, the edges start to blur and the curves become concave instead of convex when the process is continued.

To improve accuracy and to come closer to the theoretical lens geometry and aspect ratio work has to be done to avoid blurring of the edges due to the long wings of the ion beam distribution and to adapt the dwelltimes individually per milling point, considering the already milled points in the nearest neighborhood. In addition, redeposition has to be considered in the milling strategy. 

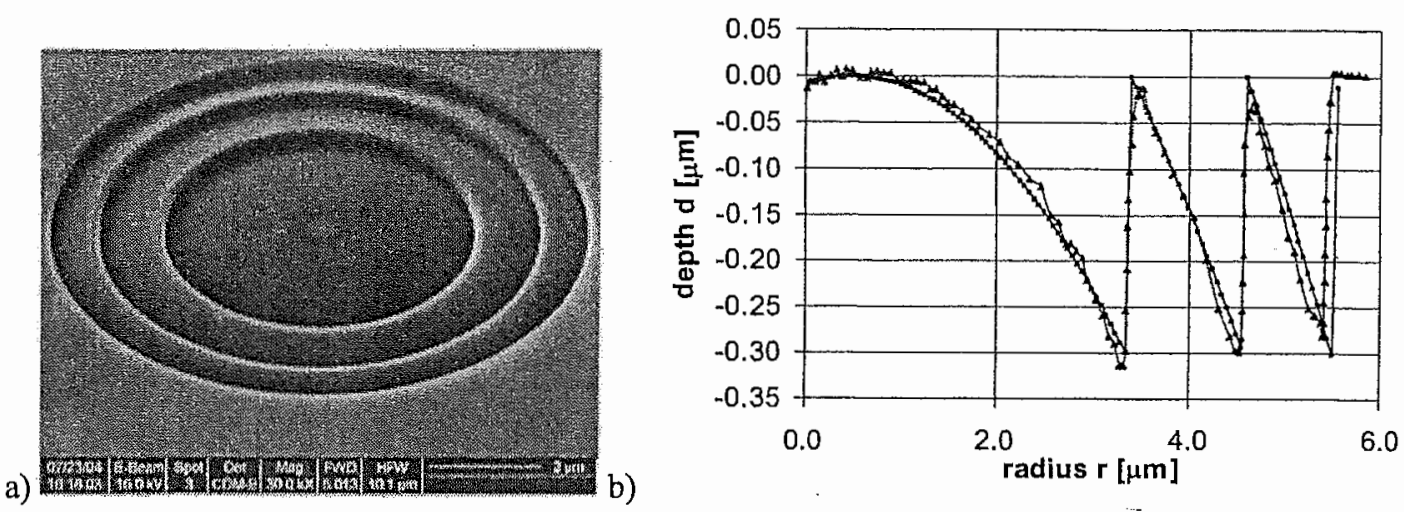

Fig. 2. Fresnel structure 2: a) overview, b) comparison with calculation (theoretical curve is scaled down $6 \mathrm{x}$ ).

\section{Photonic and sensor applications: apertures}

Cleaved fiber tips were sputtered with a gold coating of $50 \mathrm{~nm}$ thickness. For the wavelength range of interest (around $840 \mathrm{~nm}$ ) the fiber guides only one mode. In the center of the core region various apertures were patterned, i.e. the gold was milled down to the glass by the FIB. Transmission is zero except in the milled regions (apertures) which are illuminated by the guided mode.

In Fig. 3a one pattern with 9 milled squares of length $1.8 \mu \mathrm{m}$ (period $3.6 \mu \mathrm{m}$ ) is shown as an example (SEM picture). The pattern was optically characterized first in the imaging mode: with help of a microscope objective (NA $=0.65$, magnification 60) the surface of the fiber tip was brought into focus and depicted on a camera first, and then the light was switched on. The mode of an unpatterned fiber was fitted with a Gaussian curve $(\sigma=1.478 \mu \mathrm{m})$ in good approximation. The intensity distribution of the light coming out of the milled pattern was also found to be Gaussian, however shaded by the non-milled parts (Fig. 3b). This measured intensity distribution is given in linear and logarithmic scale in Fig. $3 \mathrm{~b}$ and Fig. 3c, respectively (each shows a square of 10 by $10 \mu \mathrm{m}^{2}$ ).
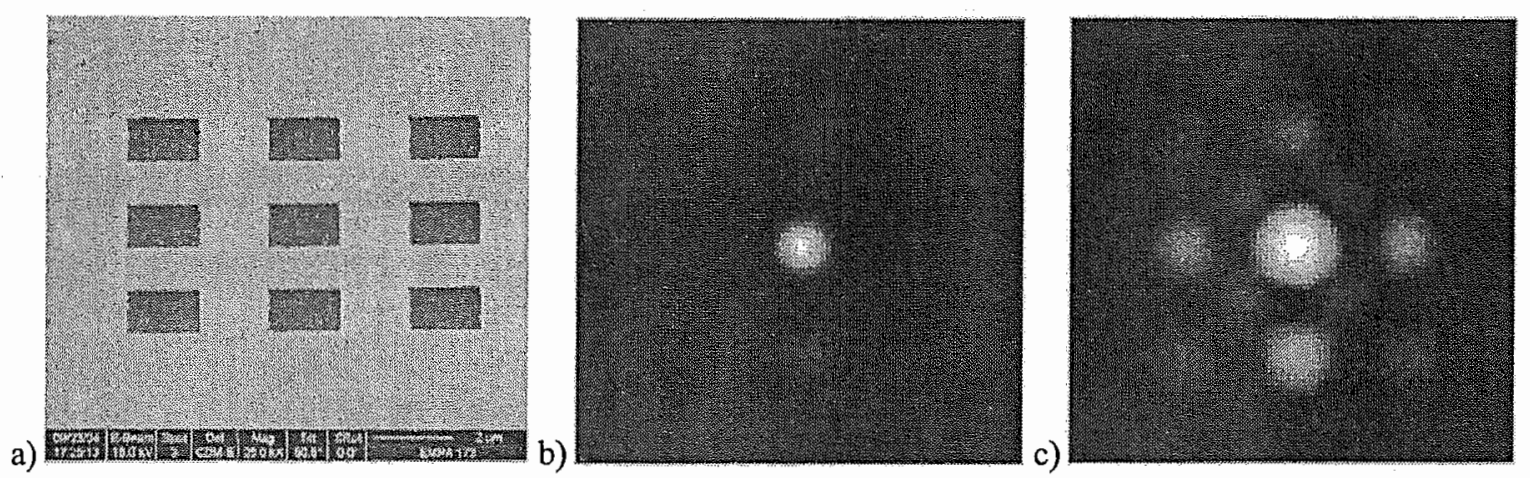

Fig. 3. $3 \times 3$ pattern milled in gold on a fiber tip (a), linear and logarithmic intensity of transmission through pattern (focus on surface of fiber) $(b, c)$.

In a next step, the far field of the sample was measured, i.e., the light emitted from the fiber tip was projected directly onto the camera without a lens and the diffraction pattern was measured in the Fraunhofer-regime. To determine the diffraction angle, the distance of the camera was varied. The result in Fig. 4 shows the increase in distance of the outer lobes in vertical and horizontal direction with increasing distance of the camera from the fiber tip. We found a diffraction angle $\alpha$ with $\tan \alpha=0.24$, which is in good agreement with the theoretical simulations. Simulations were done by Fourier transforming the transmission function of the fiber tip. The transmission function is composed of the pattern function and the mode distribution of the fiber. A measured diffraction pattern is shown in Figs. $5 \mathrm{a}$ and $\mathrm{b}$ in linear and also logarithmic scale, respectively, to improve the visibility of the faint side lobes. The corresponding simulation is given in Figs. $5 \mathrm{c}$ and $d$ and shows a good agreement. Field of view corresponds to $\tan \beta= \pm 0.4$ with $\beta$ being the angle between the fiber axis and the propagation direction. 


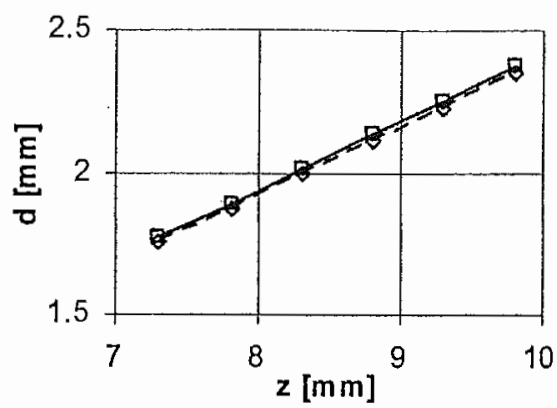

Fig. 4. Determination of diffraction angle in horizontal and vertical direction (squares and diamonds).

a)
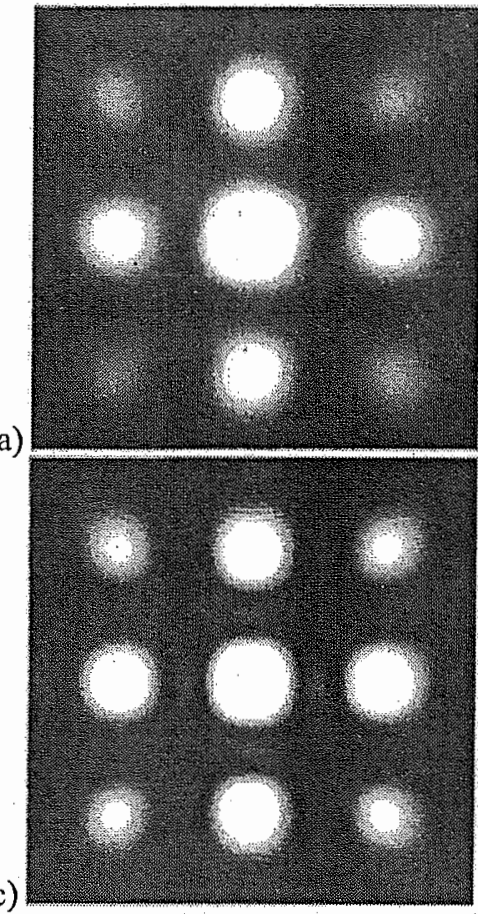

b)
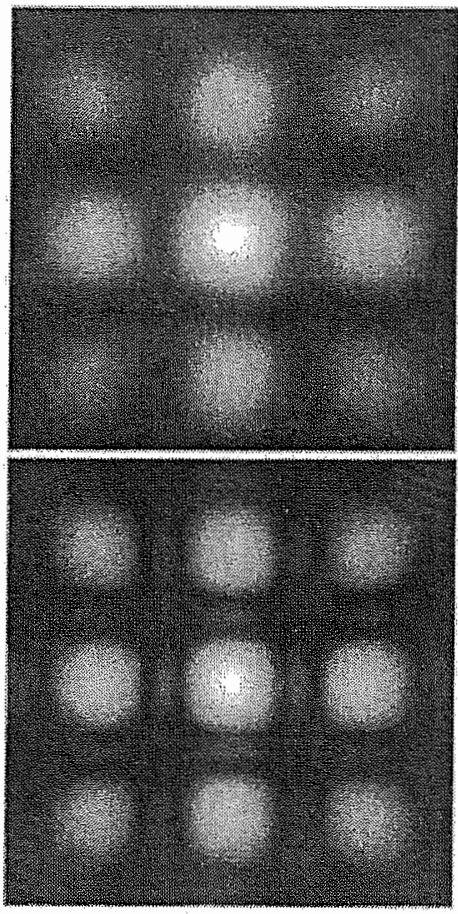

Fig. 5. Measured diffraction pattern in far field in linear (a) and logarithmic scale (b) and corresponding simulation patterns (c, d).

\section{Summary}

Promising results in FIB microstructuring of photonic elements were presented. Fresnel structures and apertures on optical fiber tips were milled and were shown to be in good agreement with theory. On the basis of these and additional experiments better modeling of the milling process will be possible and will lead to higher accuracy in FIB structuring.

\section{Acknowledgements}

The authors would like to acknowledge the discussions with and help of Andreas Beu, Stephan Meier, and Pascal Jud.

\section{References}

[1] J. Orloff, M. Utlaut, L. Swanson, High Resolution Focused Ion Beams - FIB and its Applications, Kluwer, 2003.

[2] A Tseng, Recent developments in micromilling using focused ion beam technology, topical review, J. Micromech. Microeng. 14: R15-R34, 2004.

[3] Ph. M. Nellen, R. Brönnimann, J. C. Reiner, S. Meier, A. Beu, Focused Ion Beam Microstructuring, OberflächenPolysurfaces 2005, accepted. 


\title{
PROCEEDINGS OF SPIE
}

\section{7th International Conference on Optical Fibre Sensors}

\author{
Marc Voet \\ Reinhardt Willsch \\ Wolfgang Ecke \\ Julian Jones \\ Brian Culshaw \\ Editors
}

23-27 May 2005

Bruges, Belgium

Organised by

I.D. FOS Research (Belgium)

Published by

SPIE-The International Society for Optical Engineering 